\title{
Virtual Training Environments in a Printing Company
}

\author{
Pavel Pokorný ${ }^{1}$ and Michal Birošík ${ }^{2}$ \\ ${ }^{1}$ Department of Computer and Communication Systems \\ Tomas Bata University in Zlín, Faculty of Applied Informatics \\ Nad Stráněmi 4511, 76005 Zlín, Czech Republic \\ email: pokorny@utb.cz; OCRID: $0000-0 \odot \odot 2-7393-4678$ \\ ${ }^{2}$ Department of Informatics and Artificial Intelligence \\ Tomas Bata University in Zlín, Faculty of Applied Informatics \\ Nad Stráněmi 4511, 76005 Zlín, Czech Republic \\ email: michal.birosik@gmail.com
}

\begin{abstract}
Although Virtual Reality technology has existed for decades, it has currently been experiencing a boom. This is - in particular, ensured by significant improvements in the availability and quality of Virtual Reality (VR) hardware that this technology requires. Virtual Reality is quickly expanding in many fields of human interests - scientific visualisation, the military, entertainment, healthcare, construction, etc. It is also increasingly being used in education and training. With the help of VR, industrial companies can train their employees to work more effectively, to improve their performance or to perform safe training programmes in high-risk scenarios. This paper sets out to describe proposed and implemented solutions for the use of VR for the new employee training process in a printing company. This solution includes the choice of a suitable VR headset and corresponding software tools. After that, the necessary graphical assets were prepared - (e.g. 3D models and their textures); these were then imported into the software in which the VR application was created. Its functionality was then tested in our laboratory and will be used in the relevant industrial environment.
\end{abstract}

Keywords: Virtual Reality, Modelling, Visualisation, Application

\section{Introduction}

The term Virtual Reality (VR) represents the computer-generated simulation of a 3D environment, which seems very real to the person experiencing it, using special electronic equipment. Its main objective is to achieve a strong sense of being present in the virtual environment. Because the user needs to view stereoscopic 3D scenes, today's VR technology involves wearing a head-mounted display (headset). [1]

VR is based on five key elements - a virtual world, immersion, interactivity, as well as participants and creators [2]. A Virtual World represents an imaginary space that describes a group of objects in a given space and relationships among them. Immersion means the possibility of perceiving something besides the non-physical (normal) world. Interactivity is the virtual world's response to users' action. These responses are generated in order for a user to feel fully immersed. The participant is a very important 
element because everything that happens in the VR world, happens in their mind. Hence, every Virtual Reality experience is different for each person- These experiences are given to creators - (one person or team), who design and create the virtual reality application.

Although consumer VR is only a few years old and was primarily used in the computer gaming field; over time, we are already beginning to see a staggering range of applications in many other areas of human activities. Developers are trying to build everything in the VR field. Understandably, with mixed success so far [3]. Currently, there are several domains in which VR finds applications; these include engineering, the military, robotics, architectural design, entertainment and leisure, tourism, as well as medicine, education and training, and many more.

The use of VR in education and training represents high potential. The highest motivation for using head-mounted displays is that a student/person can be exposed to various educational situations that allow them to repeatedly practice new experiences and skills in an environment that enables their correction and non-dangerous failure. Moreover, research shows that learners are generally very positive about using headmounted displays - although there are still substantial barriers to their use, especially in regards to cyber-sickness symptoms, the technical limitations of peripheral devices and the lack of appropriate software. [4] [5]

This paper deals with the proposed and implemented solution of the use of VR in a printing company for the new employees training process. Employees are one of the key factors in the development of a company - and therefore, it is important to improve their skills and their further development in order to ensure the operation of an efficient production system. New and existing employees expect to be trained, retrained and educated in the technologies and manufacturing processes used so as to understand the system, while gaining practical experience. The company's management team expect quick and effective training - which will not be unduly expensive and will not reduce the production process' profitability, efficiency and profitability. The use of Virtual Reality in this situation thus seems appropriate and advantageous.

\section{Description of the Printing Technology}

The core of the production process is a GALLUS RCS330 printing machine. In addition to the operation of the printing machine itself, the complete production process also requires the operation of other components and devices. In order to attain successful printing, the process and procedure also require the implementation of the necessary decisions and steps from print preparations to the setting of the printing machine. The following lines briefly describe the work of the operator - which was realised using Virtual Reality.

The production process begins with preparation. This consists in decision-making activities; which components should be used including the appropriate size of the printing cylinders. The production operator goes to the first device - the stand, where the printing cylinders are stored. The cylinders differ in circumference. The production op- 
erator chooses a suitable cylinder according to its size. The requirement is that the cylinder should print a surface with a single turn. The production operator takes the suitable printing cylinder and transfers it to another device - the first machine.

This machine glues the printing polymers onto the printing cylinder (Figure 1). An essential task in the printing process is the transfer of the required colour onto the printing material. The size of the printing polymer is chosen by the production operator based on the size of the order and the size of the printing cylinders. The polymer gluing device works on the principle of inserting a printing cylinder into the space between two arms. Subsequent pressing of a button activates the hydraulic piston, which closes the inserted cylinder between the arms.

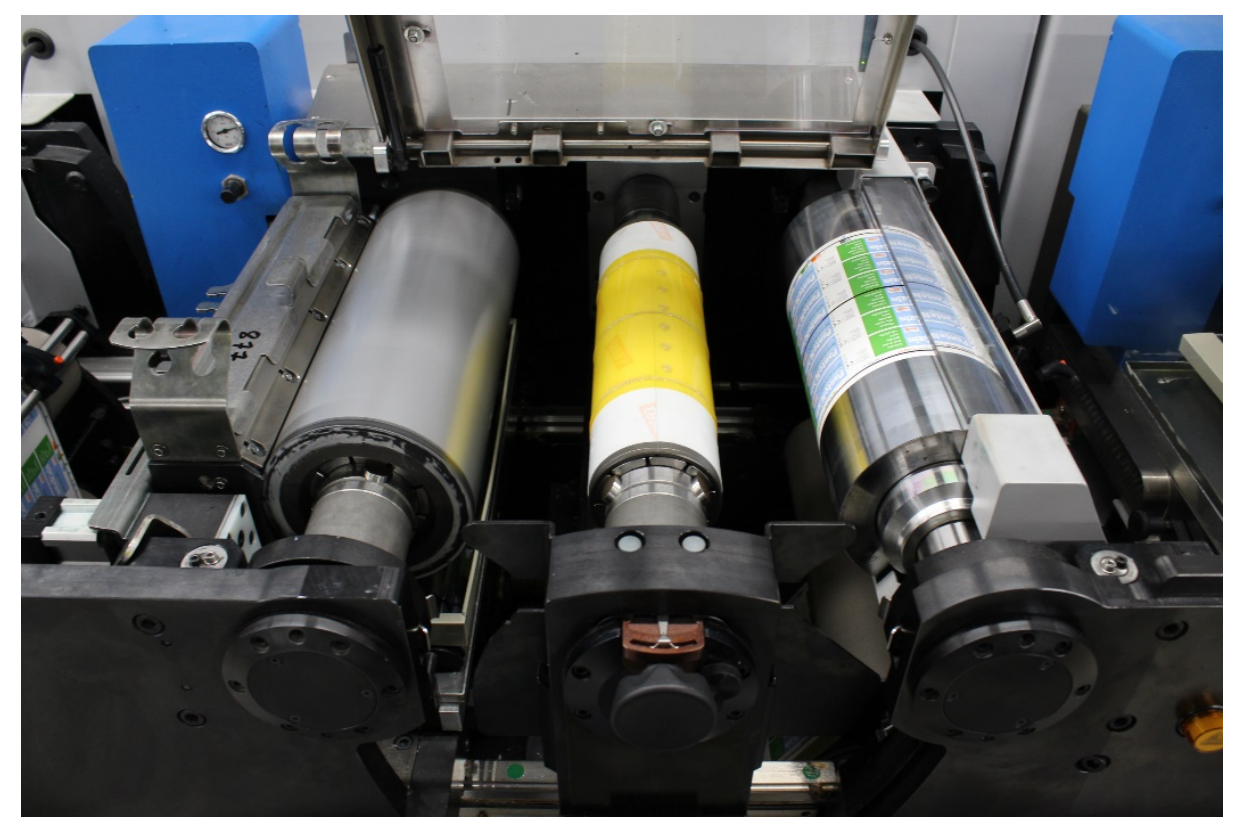

Fig. 1. The machine that glues the printing polymers on the printing cylinder

The polymer gluing process proceeds progressively from the initial application, alignment and gluing of the upper starting side. The operator checks the correctness of the initial gluing. If the polymer has not adhered correctly, the glued part is peeled off and the procedure is repeated from the beginning. If the polymer is glued correctly, the operator starts the rotation of the arms, which initiate the inserted printing cylinder's rotation. This rotation ensures that the rest of the printing polymer is glued. When this process is finished, the operator removes the printing cylinder with the glued printing polymer, releases it from the arms and goes to the printing machine with this cylinder.

This machine is used for the actual printing of patterns onto the printing material. The printable material is stored in its input hopper, which - after the start of the printing 
process moves through the individual machine parts to the printing head. The head assures the printing of parts of the final print pattern in one specific colour. The operator puts the polymer printing cylinders onto the rotating arm and secures them with screws.

The operator must also make the selection of the correct ink cartridge. Therefore, they must go to the shelf where filled and prepared ink tanks are stored, take a selected tank and return with it to the printing machine. They put the ink tank into the specified place of the printing unit of the printing machine.

Before the printing process starts, the printing material must be also put in the input part of the machine. Its type and size is chosen based on the requirements in the order.

Finally, the operator visually checks that everything is prepared correctly and goes to the printing machine control unit. Here, they activate the printing and inking (raster) component, which ensures the contact of the individual cylinders. They then check the machine once again, verify the completeness of all the previous steps of the process and starts the printing process.

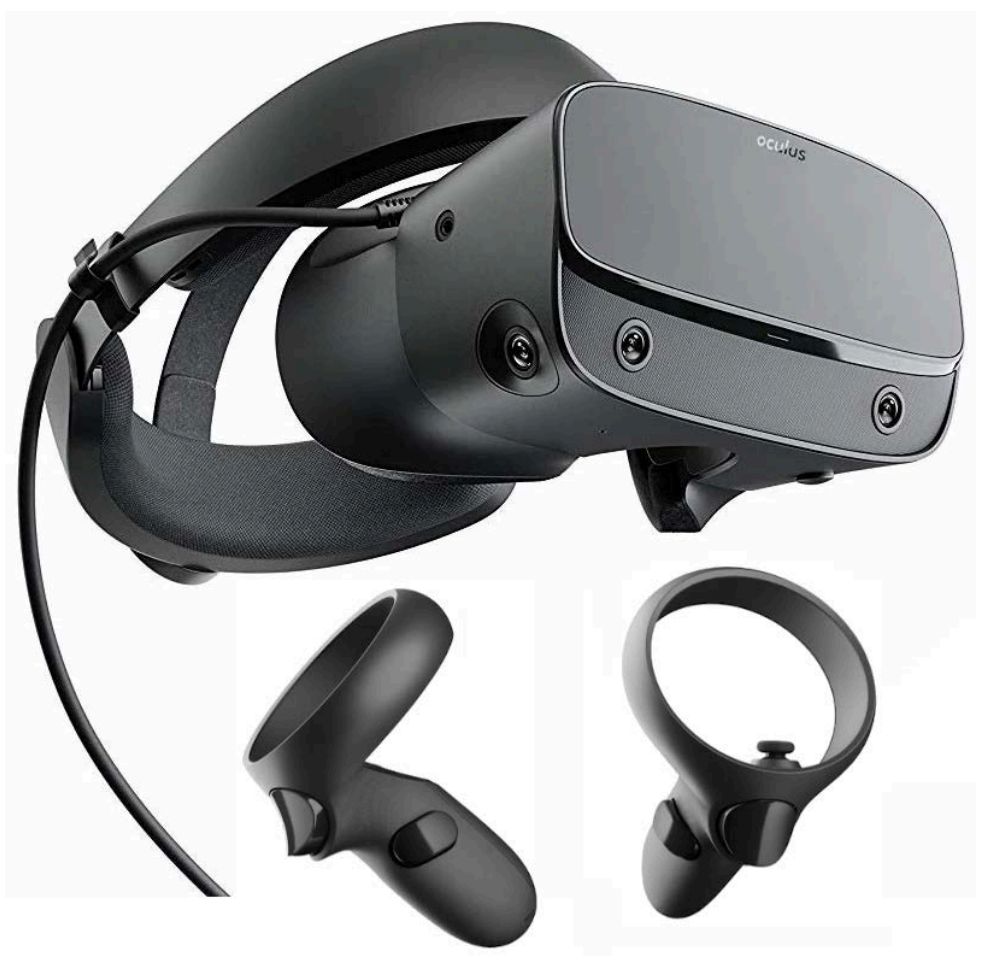

Fig. 2. An Oculus Rift Head-mounted display with its controllers

\section{The Suggested Solution}

This chapter will presented the whole suggestion of the solution itself. This includes the selection of the individual hardware and software solutions needed to create and run 
the planned VR applications. The main focus is on the choice of the suitable VR hardware, since the performance and quality of VR is essential for the final VR application and its practical use. Choosing the right software can speed up the preparation of partial 3D models, their textures and the final application.

\subsection{Hardware}

In order to create the VR application, it was decided to use a desktop form of Virtual Reality. The advantages of this solution include the fact that it is the most powerful form of VR, including price and efficiency in the use of hardware devices. There are a number of manufacturing companies that are occupied with desktop forms of Virtual Reality technology.

Based on the requirements, Oculus Rift (Fig. 2) was finally selected because it offers a combination of quality and powerful technologies that promise VR at a high level. It uses the OLED display technology, supports 2160 x 1200 resolution, $90 \mathrm{~Hz}$ refresh rate and 110 degrees of (diagonal) field-of-view. It also includes an integrated audio VR that provides 3D audio effects, and positional tracking is accomplished with the help of infrared sensors that allow one to use it while standing, sitting or walking around the room. This device is compatible with the Microsoft Windows platform, and the possible development of other applications for this device. [6]

\subsection{Software Tools}

In order to create the planned VR application, three software tools were used in their latest versions - Blender, Substance Painter and Unity. Blender was used to perform the preparation of all necessary 3D models with their corresponding UV maps. Then, Substance Painter was used to create all textures. All models and their textures were then imported into the Unity engine. Unity was used as the developer environment, in which the final application was programmed.

Blender is a free and open-source 3D creation suite. It supports the entirety of the 3D pipeline - modelling, rigging, animation, simulation, rendering, compositing and motion tracking - and even allows video editing and game creation [7]. It provides all the requisite tools for creating models and scenes that may be made into still images or animated movies. In order to attain effective work, Blender supports a wide variety of graphic tools. The created models and scenes can then be exported into many graphic formats - including images (jpeg, jpeg2000, png, tga, tiff), video (avi, mpeg, quicktime) and 3D formats (3ds, dae, dxf, fbx, obj, stl, svg, vrml, x3d), and many others. [8]

Textures were created in the Substance Painter suite [9]. This tool encapsulates all necessary commands for the texturing of 3D assets. It supports smart materials and smart masks that are adapted to a user's models, performs real-time painting in 3D space using smart brushes, projection tools and even particles, fast renderer, extracting and baking any material from any 3D object with a GPU accelerated baker, imports assets from many commonly-used graphic formats and then exports them to any game engine. [10]

Unity [11] is a cross-platform real-time engine that provides an excellent entry-point into game and 3D application development, balancing features and functionality with 
price point. The free Unity version allows people to experiment, learn, develop, and sell games before committing any of their hard-earned money. Thanks to its huge user community, Unity removes the elitist separation between programmers, game designers and artists that is typical of high-priced game engines. It makes it possible for anyone to take the first step to bringing their ideas to life [12]. The Unity engine offers primary scripting in the $\mathrm{C \#}$ programming language, but many scripts are prepared in templates and are easier to use without a deeper knowledge of this programming language. [13]

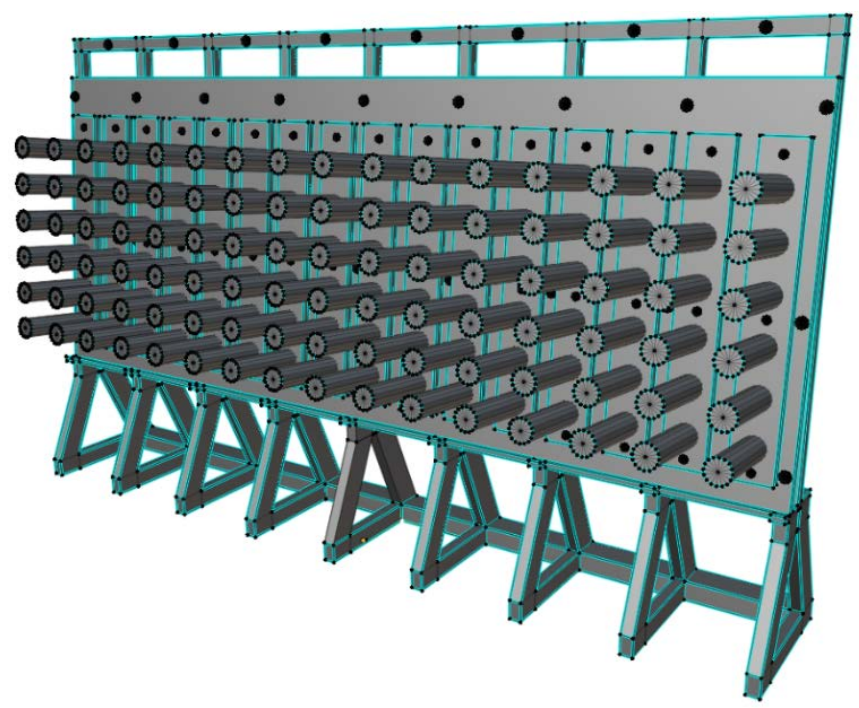

Fig. 3.The 3D Model of the Printing Cylinder Stand

\section{$4 \quad 3 D$ Models and Their Textures}

All models created in Blender use polygonal representation - a collection of vertices, edges and faces that define the geometric shapes of objects. Preference was given to the so-called box modelling process for model creation. This is a technique, where primitive shapes are used to begin with - (Planes, Circles, Cubes, Tubes, Spheres, etc.) and these objects are then shaped to the final model using standard modelling tools. Blender offers many editing, transformation and modelling tools and these then often generate new vertices, edges and faces so they allow one to make more detailed and better shaped models. Examples of these tools are Extrude, Bevel, Loop Cut, Knife, Subdivide or Insert Face [14]. Figure 3 shows the model of a printing cylinders stand which was modelled in this way.

Should one need a rounded shape, Blender performs this in the Sculpt Mode. This mode is used for shape-forming, where brushes manipulate an object's whole geometry in the brush region of influence and shapes it based on the dependency of brushes type and its set parameters. 


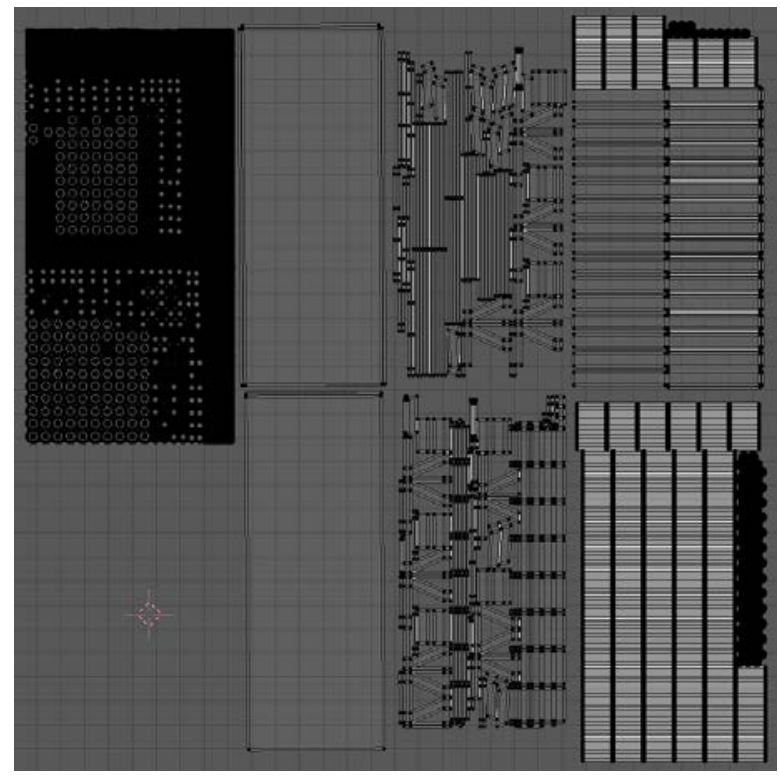

Fig. 4. UV Map for the Stand from Fig. 2

When the shapes of all mesh models have been created, the UV mapping phase is started. The main goal in this phase is to make UV maps for all of them and their entire faces. In order to obtain UV maps, Blender supports the Unwrap command. Before this command runs, it is necessary to mark the edges of the modelled objects, where the faces must be ripped in order to obtain the correct 2D image. If the UV maps do not correspond to our ideas - (all UV maps should cover image areas as much as possible), they are improved manually. An example of such a UV map is shown in Fig. 4.

When the modelling and UV map have been completed, all created models are then exported via the .fbx graphic format and the UV maps then exported in the tiff graphic format with a $1024 \times 1024$ pixel resolution.

All textures were drawn in the Substance Painter application. The technique Relief Mapping was used, because it performs accurate and effective render surface detail of 3D objects. Depending on what texture affects - (colours, transparency, bumps, glossy, etc.), they were then separated into multiple layers. An example of the draw colour layer for the UV map in Fig. 4 is shown in Fig. 5. The final appearance of the surface was specified by a combination of multiple layers of materials or textures.

When all of the textures for the 3D models had been created, they were exported to the Unity engine. Their resolution was increased to 4096 x 4096 pixels in order to attain the very high quality of 3D objects later in the Virtual Reality application and the output texture format was set to .tiff. 


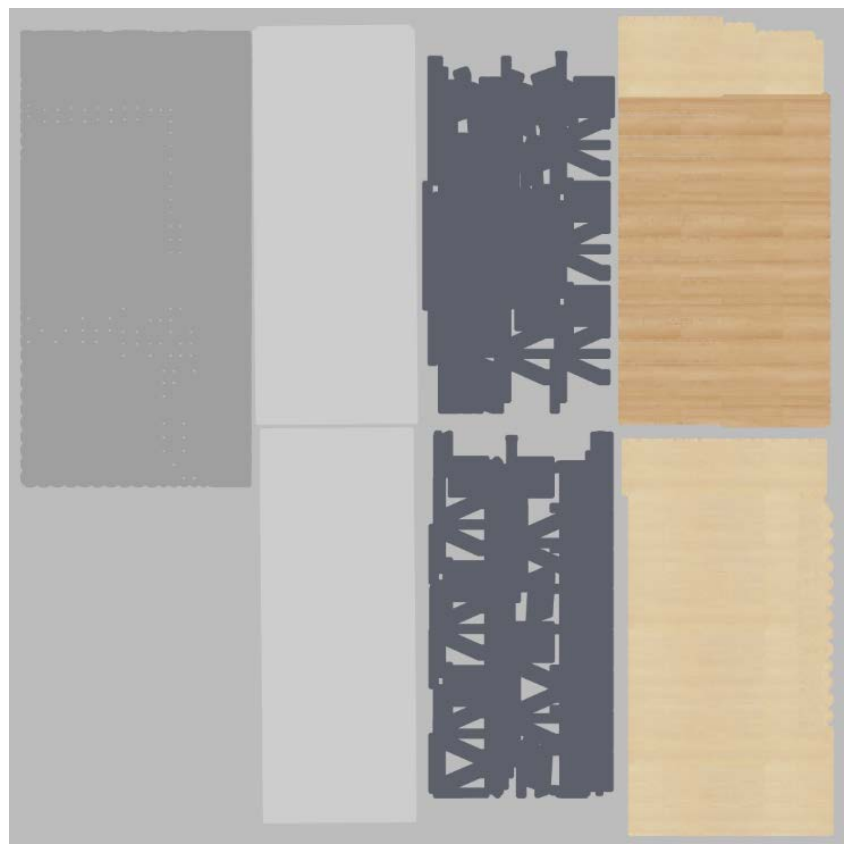

Fig. 5. UV Texture for the Stand from Fig. 2 (Substance Painter Output)

\section{$5 \quad$ An Application}

When all graphic assets had been prepared, the next step was to install drivers and the necessary software for Oculus Rift. The requisite Oculus Rift Software installation package was downloaded from the manufacturer's website [15], and then installed. Since we also wanted to use some sounds in our application, the requisite sounds in .mp3 format were downloaded from the website [16]. We planned to use sound effects for the user-interface (select commands from menus, button press, button hover), as well as sounds reproducing walking, running, grabbing, dropping the object, and the sounds of the printing machine while the printing process runs. The appropriate sounds for these actions were downloaded.

Then a new 3D project was created in the Unity engine. The Oculus Integration asset package was added during this creation process in order to have Oculus device support in this project. The next step was to add all prepared assets - (i.e. 3D models, textures, sounds) to this project. After that, the 3D models - with their textures, were placed in the scene. They were transformed - (moved, rotated or scaled) if necessary. The whole VR environment was made in this way. Fig. 6 shows the model of the stand with printing cylinders in this phase. 


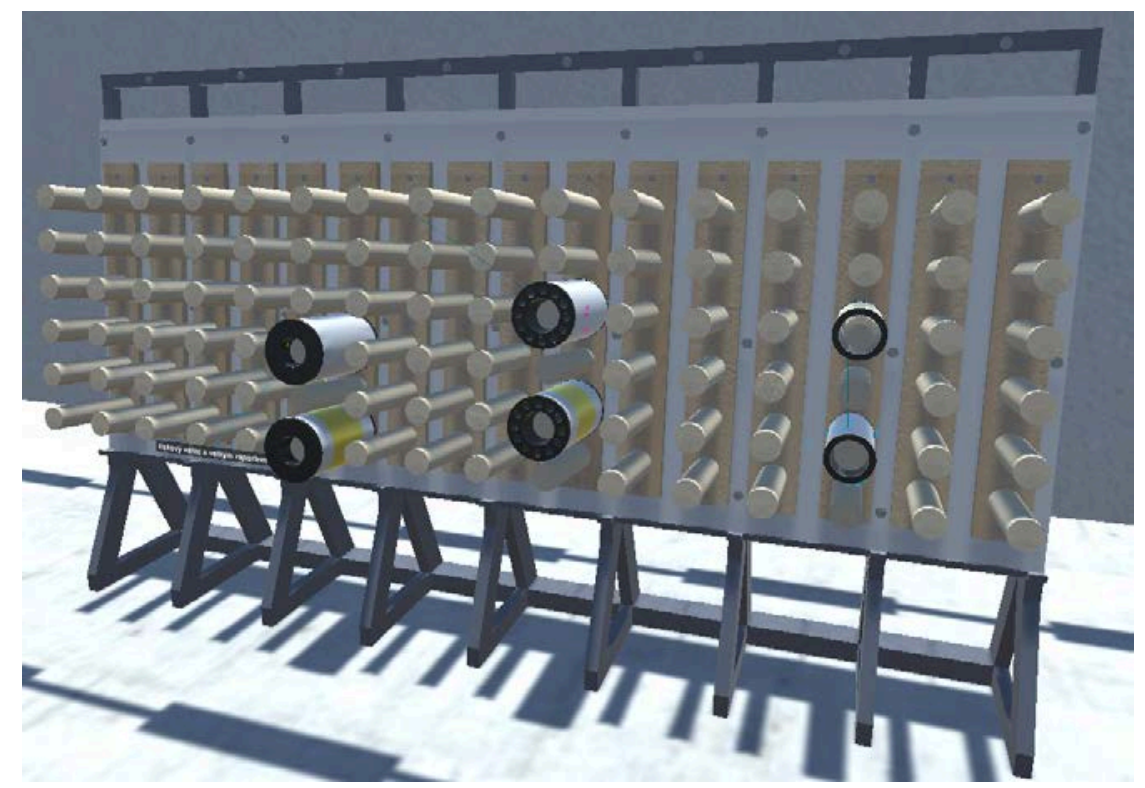

Fig. 6.The Stand with Mapped Textures in the Unity Engine

This process was then augmented by the addition of colliders - (simple objects like boxes), which were provided by Unity itself. These were added to all added 3D models and their individual components. Then, colliders that serve as sensors were added in order to perform the running of programmed functions in the event of a collision with another collider in the environment. It was also necessary to add objects based on the user's choice to allow one to highlight the requisite space or object in the scene. The Unity ParticleSystem object was used for this purpose.

A control panel was the last physical object created in this phase. This implies the need for buttons for operating the device for the printing polymer. The buttons were then animated - (using the Animator component in Unity), in order to simulate their pushing. Then, sounds were added to the appropriate objects and their properties set (e.g. volume, situations in which they should be played, if they are to be played in cycles, etc.).

Before the application was first tested, it was necessary to insert the most important VR objects into the scene hierarchy. Mainly, this includes the OVRPlayerController object - which ensures the whole functionality of all VR devices - (i.e. capturing and tracking the position of the headset, capturing controls, controlling character movement and representing the main camera). CustomHandLeft and CustomHandRight were other necessary VR objects; these represent virtual hands - (communication tools between the user and virtual environment). These hands provide base functionality via scripts and were set as necessary in this application.

After the first testing stage and the elimination of minor mistakes, the creation of the user interface - (main menu, settings, control of devices, etc.) began. Its base is built on the Canvas object that can contain sub-objects like Quads, Panels or Buttons. These 
individual items were then set up for interactions with the user, whose interventions can run the created scripts. Text objects to characterise corresponding commands have been added as needed. All texts displayed in the developed VR application were written in the Czech language, since this application was developed for a Czech manufacturing company.

Since our VR application was focused on the new employee training and education processes, the scene was further expanded by auxiliary objects labelled with simple text descriptions that simply describe the individual machine parts and their purposes. Fig. 7 shows the printing machine with these text descriptions in the Unity engine.

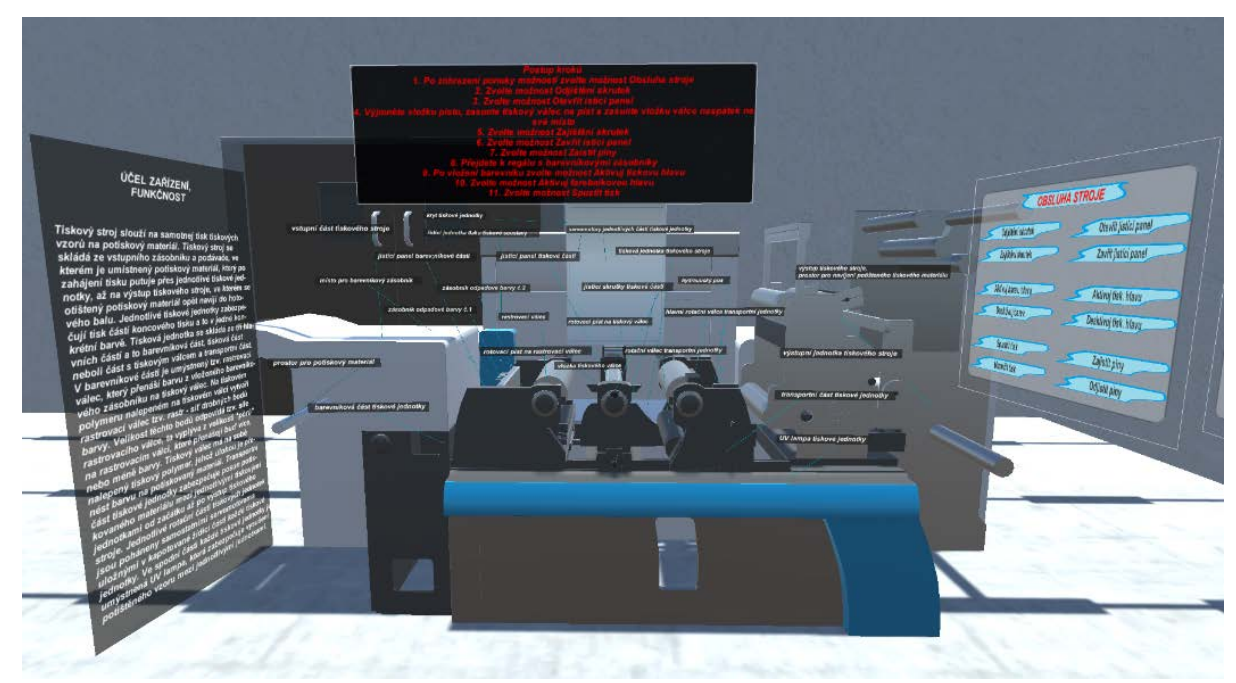

Fig. 7. The Printing Machine in the Unity Engine with Descriptions and Instructions (in Czech)

With these activities completed, the 3D scene was finished and was ready to create the application logic. This included the creation of individual devices scripts and functionalities, by user interactions. Some scripts were already integrated (the basic motion control, headset interaction with the scene, sound playback). The missing scripts were written directly in Unity; and programme source codes were then created in the C\# programming language in the Microsoft Visual Studio 2017 environment.

After creating all parts of the application - including application logic and testing its functionality, the standalone VR application was built. The target platform was set into the Windows x86_64 architecture. The application was tested under laboratory conditions and some bugs that appeared during its development were fixed.

\section{Conclusion}

This paper describes the use of VR for the new employee training process in a printing company environment. Current research shows that its use in the education and training processes offers huge potential. The benefits can include lower costs, the acceleration 
of training, better quality - (it is possible to learn in a natural "trial and error" way), its attractiveness, simplification - (the reduction of the cognitive complexity of training), and streamlining - (the possibility of creating simulations and training according to their own ideas and needs and further possible expansions or modifications of the same). The shortcomings include the possibility of cyber-sickness symptoms and technical limits of the hardware devices.

The main outcome of this work is a fully functional VR application that simulates the printing production process. The most difficult - and longest part of the work, was the most accurate creation of all 3D models, the preparation of their UV maps, and the texturing process during the creation of the VR application. This application is fully functional and capable of being implemented into the real production process. It also provides feedback that will allow the created application to be optimised/improved and should also prove the success of the use of VR in this area.

In the future, it would be possible to expand the application with a detailed layout and analysis of individual machines and their parts, with the addition of animations and information about the most common faults. This could also essentially complement the application with another way to use VR - as a service manual. The application could also be extended in order to simulate other production processes and their procedures in this manufacturing company - or other manufacturing companies.

\section{References}

1. Linowes J.: Unity Virtual Reality Projects. 2nd edn. Packt Publishing, Birmingham (2018). ISBN 978-1-78847-880-9.

2. Sherman, W.R., Craig, A.B.: Understanding Virtual Reality: Interface, application and design. Morgan Kaufmann, Cambridge (2019). ISBN 978-0-12-818399-1.

3. Parisi, T.: Learning Virtual Reality: Developing Immersive Experiences and Applications for Desktop, Web, and Mobile. $1^{\text {st }}$ edition. O'Reilly Media (2015). ISBN 978-1-49192-2835.

4. Jensen, L., \& Konradsen, F. A review of the use of virtual reality head-mounted displays in education and training. Education and Information Technologies, 23(4) (2018). ISSN: 15151529.

5. Wang, P., Wu, P., Wang, J., Chi, H. L., \& Wang, X. A critical review of the use of virtual reality in construction engineering education and training. International journal of environmental research and public health, 15(6), 1204 (2018).

6. Oculus Rift: VR Headset for Computers prepared for VR, https://oculus.com/rift-s/, last accessed 2020/01/18.

7. Blender.org - Home of the Blender project, https://www.blender.org/, last accessed 2020/01/18

8. Blain, J. M.: The Complete Guide to Blender Graphics, 3rd Edition, CRC Press (2016). ISBN 978-1-4987-4647-2.

9. Substance Painter, https://www.substance3d.com/products/substance-painter/, last accessed 2020/01/18.

10. Toudou: Substance Painter for Blender user. 1st edn. Amazon.com Services LLC (2017).

11. Unity Real-Time Development Platform, https://unity.com/, last accessed 2020/01/18. 
12. Blackman, S.: Unity for Absolute Beginners. 1st edn. Apress. (2014). ISBN 978-1-43026779-9.

13. Blackman, S., Tuliper, A. Learn Unity for Windows 10 Game Development, APress (2016). ISBN 978-1-4302-6758-4.

14. Guevarra, E.T.M.: Modeling and Animation Using Blender:Blender 2.80:The Rise of Eevee. 1st edn. Apress (2020). ISBN 978-1-4842-5339-7.

15. Oculus Setup: Rift S, Rift, Quest and Go, https://oculus.com/setup, last accessed 2020/01/18.

16. Freeound - Freesound, https://freesound.org/, last accessed 2020/01/18. 